\title{
Sum-Rate Maximization under QoS Constraint in MIMO-NOMA Systems
}

\author{
Ming Zeng, Viktoria Fodor \\ School of Electrical Engineering, KTH Royal Institute of Technology, Stockholm, Sweden \\ \{mzeng,vfodor\}@kth.se
}

\begin{abstract}
This paper addresses the power allocation challenge for the downlink transmission in non-orthogonal multiple access (NOMA) systems applying multiple input multiple output transceivers. We consider the case when users are paired to form NOMA clusters, and share a common power budget. We provide low complexity power allocation methods within the clusters and across the clusters, that, together, maximize the sum-rate of the network, while guaranteeing a minimum quality of service for the users with weak channel condition. We show that compared to equal power allocation for the clusters, the proposed power allocation scheme improves the system fairness significantly, without decreasing the aggregate performance.
\end{abstract}

\section{INTRODUCTION}

Non-orthogonal multiple access (NOMA) is recognized as one of the promising technologies to increase the spectral efficiency of mobile networks [1]-[3]. NOMA multiplexes the users in the power domain, by applying superposition coding at the transmitter, and successive interference cancellation (SIC) at the receiver. Since users are multiplexed in the power domain, power allocation (PA) affects the network performance significantly.

As proved in [4] for single-input single-output (SISO) systems, sum rate maximization under NOMA would lead to assigning all power to the user with the best channel condition, and consequently, the introduction of fair power allocation policies is necessary. Therefore, [4] proposes weighted sum rate maximization, while [5] guarantees the rates achieved by all the users under NOMA to be as good as that under conventional orthogonal multiple access (OMA). [6] introduces the concept of cognitive radio (CR) inspired PA, that considers the weak user as the primary user to ensure a minimum quality of service (QoS), and further proves that this CR-inspired power allocation can achieve higher sum rate than simply serving the weak users.

With the proliferation of multiple-input multiple-output (MIMO) antennas, it is of interest to apply NOMA for MIMO systems. The design of MIMO-NOMA downlink transmission is however challenging, since channels are not scalar as in SISO systems, and therefore it is difficult to order the users according to the channel matrix. [7] proposes a tractable solution, where users are first clustered into pairs, and then the MIMO channel is turned into multiple SISO channels with appropraite precoding and detection matrics. However, this work only applies to the case when the number of receiving antennas equals or exceeds that of the transmitting antennas. To loose this constraint, a more general MIMO-NOMA system is proposed in [8], where signal alignment is applied for the users within a cluster. CR-inspired PA is introduced in [7] and [8] as well, though only applied between users in the same cluster, while assigning equal power for each cluster. In this case, the user admission is quite simple: if the QoS of the weak user in the cluster can be satisfied, it will be admitted; otherwise an outage occurs. Clearly, by allowing power to be transferred to clusters with very weak users, more users could be admitted, and the the sum rate of the system could be further improved.

Inspired by this intuition, in this paper we propose crosscluster optimized PA. We consider that the users are already paired to clusters, and each weak user has a QoS constraint as [6]-[8]. We define the system objective as follows: if the total transmit power can support all weak users, the objective is to maximize the sum rate of all the users, while fulfilling the QoS constraints of the weak users. Otherwise, the objective of the PA is to maximize the number of admitted weak users. For the first case, we propose C-NOMA, an iterative, cross-cluster sum rate maximization algorithm and prove its optimality. We also provide an optimal admission algorithm, when not all weak users can be supported. Via numerical results we show that the proposed C-NOMA can halve the outage probability of the weak users compared to the CR-inspired PA in [7], without decreasing the sum rate of the system. Moreover, CNOMA increases the fairness among the clusters as well as among the individual users in the low power regime.

The rest of the paper is organized as follows. The system model and problem formulation are introduced in Section II. The proposed PA scheme is presented and analyzed in Section III, while simulation results are shown in Section IV. Conclusions are finally drawn in Section V.

\section{System Model and Problem Formulation}

\section{A. System Model}

We consider a downlink multiuser MIMO system in this paper, where the base station (BS) with $M$ antennas transmits data to multiple receivers, each with $N$ antennas. The total number of users in the system is $2 M$, and users are paired into $M$ clusters with two users per cluster. The wireless channels between the BS and users are characterized by quasi-static Rayleigh fading and large scale path loss. Particularly, the channel matrix for user $(m, k), m \in\{1, \cdots, M\}, k \in\{1,2\}$ is $\mathbf{H}_{m, k}=\frac{\mathbf{G}_{m, k}}{L_{m, k}}$, where $\mathbf{G}_{m, k} \in \mathbb{C}^{N \times M}$ denotes the Rayleigh 
fading channel matrix, while $L_{m, k}$ represents the path loss component.

The system adopts signal alignment within the cluster and zero forcing precoding among the clusters to avoid intercluster interference, as proposed in [8]. Moreover, SIC is employed within each cluster.

Specifically, the $M \times 1$ information-bearing vector transmitted from the BS is given by

$$
\mathbf{s}=\left[\begin{array}{c}
\sqrt{P_{\max } \Omega_{1,1}} s_{1,1}+\sqrt{P_{\max } \Omega_{1,2}} s_{1,2} \\
\vdots \\
\sqrt{P_{\max } \Omega_{M, 1}} s_{M, 1}+\sqrt{P_{\max } \Omega_{M, 2}} s_{M, 2}
\end{array}\right],
$$

where $s_{m, k}$ is the signal intended for user $k$ in cluster $m, \Omega_{m, k}$ is the corresponding power coefficient, satisfying $\sum_{m=1}^{M}\left(\Omega_{m, 1}+\Omega_{m, 2}\right) \leq 1$, and $P_{\max }$ is the maximum transmit power.

For any user $(m, k)$, the received signal can be expressed as

$$
\mathbf{y}_{m, k}=\mathbf{H}_{m, k} \mathbf{P s}+\mathbf{n}_{m, k}
$$

where $\mathbf{P}=\left[\mathbf{p}_{1}, \cdots, \mathbf{p}_{M}\right] \in \mathbb{C}^{M \times M}$ denotes the precoding matrix, while $\mathbf{n}_{m, k} \in \mathbb{C}^{N \times 1}$ is the noise vector modelled as independent and identically distributed (i.i.d.), $\mathcal{C N}\left(0, \sigma_{n}^{2} \mathbf{I}\right)$.

By applying the detection vector $\mathbf{v}_{m, k} \in \mathbb{C}^{N \times 1}$ on the received signal, we get

$$
\begin{aligned}
& \mathbf{v}_{m, k}^{H} \mathbf{y}_{m, k} \\
& =\mathbf{v}_{m, k}^{H} \mathbf{H}_{m, k} \mathbf{p}_{m}\left(\sqrt{P_{\max } \Omega_{m, 1}} s_{m, 1}+\sqrt{P_{\max } \Omega_{m, 2}} s_{m, 2}\right) \\
& +\underbrace{\sum_{l=1, l \neq m}^{M} \mathbf{v}_{m, k}^{H} \mathbf{H}_{m, k} \mathbf{p}_{l} \mathbf{s}_{l}}_{\text {interference from other clusters }}+\mathbf{v}_{m, k}^{H} \mathbf{n}_{m, k},
\end{aligned}
$$

where $\mathbf{s}_{l}$ denotes row $l$ of $\mathbf{s}$.

Due to zero forcing precoding $\mathbf{v}_{m, k}^{H} \mathbf{H}_{m, k} \mathbf{p}_{l}=0$ for any $l \neq m$, the above equation can be simplified as

$$
\begin{aligned}
& \mathbf{v}_{m, k}^{H} \mathbf{y}_{m, k} \\
& =\mathbf{v}_{m, k}^{H} \mathbf{H}_{m, k} \mathbf{p}_{m}\left(\sqrt{P_{\max } \Omega_{m, 1}} s_{m, 1}+\sqrt{P_{\max } \Omega_{m, 2}} s_{m, 2}\right) \\
& \quad+\mathbf{v}_{m, k}^{H} \mathbf{n}_{m, k} .
\end{aligned}
$$

Let us now consider the users in cluster $m$, and without loss of generality, let user $(m, 1)$ have a smaller path loss than user $(m, 2)$, i.e., $\frac{1}{L_{m, 1}}>\frac{1}{L_{m, 2}}$. The signal alignment in the cluster ensures $\mathbf{v}_{m, 1}^{H} \mathbf{G}_{m, 1}=\mathbf{v}_{m, 2}^{H} \mathbf{G}_{m, 2}$ (eq. 7, [8]).

Thus, it can be easily obtained that

$$
\left|\mathbf{v}_{m, 1}^{H} \mathbf{H}_{m, 1} \mathbf{p}_{m}\right|^{2}>\left|\mathbf{v}_{m, 2}^{H} \mathbf{H}_{m, 2} \mathbf{p}_{m}\right|^{2} .
$$

At the receiver side, user $(m, 1)$ conducts SIC to remove the interference from user $(m, 2)$, and thus the signal-tointerference-plus-noise ratio (SINR) of user $(m, 1)$ can be expressed as

$$
\begin{aligned}
\Gamma_{m, 1} & =\frac{P_{\max } \Omega_{m, 1}\left|\mathbf{v}_{m, 1}^{H} \mathbf{H}_{m, 1} \mathbf{p}_{m}\right|^{2}}{\mathbb{E}\left\{\left|\mathbf{v}_{m, 1}^{H} \mathbf{n}_{m, 1}\right|^{2}\right\}} \\
& =\rho \Omega_{m, 1}\left|\mathbf{v}_{m, 1}^{H} \mathbf{H}_{m, 1} \mathbf{p}_{m}\right|^{2},
\end{aligned}
$$

where $\rho=P_{\max } / \mathbb{E}\left\{\left|\mathbf{v}_{m}^{H} \mathbf{n}_{m}\right|^{2}\right\}$ is the same for all users, since the detection vector is normalized and the noise variance remains unchanged after rotation. $\mathbb{E}$ denotes the expectation operation.

Thus, its achievable rate is given by

$$
R_{m, 1}=\log _{2}\left(1+\rho \Omega_{m, 1}\left|\mathbf{v}_{m, 1}^{H} \mathbf{H}_{m, 1} \mathbf{p}_{m}\right|^{2}\right) .
$$

At user $(m, 2)$, the signal to user $(m, 1)$ is considered as interference, thus the SINR is given by

$$
\Gamma_{m, 2}=\frac{\rho \Omega_{m, 2}\left|\mathbf{v}_{m, 2}^{H} \mathbf{H}_{m, 2} \mathbf{p}_{m}\right|^{2}}{\rho \Omega_{m, 1}\left|\mathbf{v}_{m, 2}^{H} \mathbf{H}_{m, 2} \mathbf{p}_{m}\right|^{2}+1},
$$

and the achievable rate is

$$
R_{m, 2}=\log _{2}\left(1+\frac{\rho \Omega_{m, 2}\left|\mathbf{v}_{m, 2}^{H} \mathbf{H}_{m, 2} \mathbf{p}_{m}\right|^{2}}{\rho \Omega_{m, 1}\left|\mathbf{v}_{m, 2}^{H} \mathbf{H}_{m, 2} \mathbf{p}_{m}\right|^{2}+1}\right) .
$$

\section{B. Problem Formulation}

To enhance user fairness, we assume that the weak user in each cluster has a QoS requirement, i.e., $R_{m, 2}^{\min }$, that needs to be ensured according to the $\mathrm{CR}$ principle. The system objective is then to maximize the sum rate under the QoS requirement for the weak users. The considered problem can be formulated as:

$$
\begin{array}{ll}
\max _{\Omega_{m}, l} & \sum_{m=1}^{M} \sum_{l=1}^{2} R_{m, l} \\
\text { s.t. } & R_{m, 2} \geq R_{m, 2}^{\min }, \forall m, \\
& \sum_{m=1}^{M} \sum_{l=1}^{2} \Omega_{m, l} \leq 1 .
\end{array}
$$

Clearly, problem (10) is not feasible if $P_{\max }$ can not satisfy the QoS requirements of all weak users. In that case, we propose an alternative objective, to maximize the number of satisfied weak users, that is,

$$
\begin{array}{ll}
\max _{\Omega_{m, l}} & \sum_{m=1}^{M} x_{m} \\
\text { s.t. } & R_{m, 2} \geq R_{m, 2}^{\min } x_{m}, \forall m, \\
& \Omega_{m, 1}=0, \quad \forall m, \\
& \sum_{m=1}^{M} \Omega_{m, 2} \leq 1, \\
& x_{m} \in\{0,1\}, \quad \forall m,
\end{array}
$$

where $x_{m}$ is the decision variable indicating whether user $(m, 2)$ is admitted.

\section{C-NOMA Power AllocAtion}

First, we need to determine whether or not problem (10) is feasible. The required power to satisfy the weak users is minimized, if no power is allocated to the strong users in the clusters. That is, problem (10) is feasible if

$$
P_{\text {req }}=P_{\max } \sum_{m=1}^{M} \Omega_{m, 2}^{\min }
$$


where, according to (9), $\Omega_{m, 2}^{\min }$ is given by

$$
\Omega_{m, 2}^{\min }=\frac{2^{R_{m, 2}^{\min }-1}}{\rho\left|\mathbf{v}_{m, 2}^{H} \mathbf{H}_{m, 2} \mathbf{p}_{m}\right|^{2}} .
$$

As a result, all weak users can be admitted if and only if

$$
P_{\text {req }} \leq P_{\max } .
$$

A. C-NOMA for sum rate maximization when (10) is feasible

Investigating problem (10), (10b) is clearly a convex constraint, and (10c) is an affine function over the power coefficients. However, due to the interference introduced by signal superposition, the objective function (10a) is non-convex. Consequently, no standard convex optimization methods can be applied to solve the maximization problem. In the following, we propose an optimal PA solution, which exploits the properties of the NOMA-based system.

We address the maximization problem in the clustered NOMA system in two steps. First we derive how to allocate power within a cluster $m$ under given total power for the cluster. Based on this result, we propose optimal power allocation across the clusters.

For the first step, we introduce the following lemma.

Lemma 1: For a given total power constraint for a twouser cluster, the cluster sum rate is maximized if the power is allocated such that the weak user just satisfies its QoS, while the strong user gets all the remaining power.

Proof: Denote the total power constraint for cluster $m$ as $P_{m}=\beta P_{\max }, \beta \in[0,1]$. Then $\Omega_{m, 1}+\Omega_{m, 2}=\beta$ has to hold. The sum rate, $R_{m}^{\text {sum }}=R_{m, 1}+R_{m, 2}$, can be expressed as

$$
\begin{aligned}
R_{m}^{\text {sum }}= & \log _{2}\left(1+\rho \Omega_{m, 1}\left|\mathbf{v}_{m, 1}^{H} \mathbf{H}_{m, 1} \mathbf{p}_{m}\right|^{2}\right) \\
& +\log _{2}\left(1+\frac{\rho \Omega_{m, 2}\left|\mathbf{v}_{m, 2}^{H} \mathbf{H}_{m, 2} \mathbf{p}_{m}\right|^{2}}{\rho \Omega_{m, 1}\left|\mathbf{v}_{m, 2}^{H} \mathbf{H}_{m, 2} \mathbf{p}_{m}\right|^{2}+1}\right) \\
= & \log _{2}\left(1+\rho \Omega_{m, 1}\left|\mathbf{v}_{m, 1}^{H} \mathbf{H}_{m, 1} \mathbf{p}_{m}\right|^{2}\right) \\
& -\log _{2}\left(1+\rho \Omega_{m, 1}\left|\mathbf{v}_{m, 2}^{H} \mathbf{H}_{m, 2} \mathbf{p}_{m}\right|^{2}\right) \\
& +\log _{2}\left(1+\beta \rho\left|\mathbf{v}_{m, 2}^{H} \mathbf{H}_{m, 2} \mathbf{p}_{m}\right|^{2}\right) .
\end{aligned}
$$

With $\left|\mathbf{v}_{m, 1}^{H} \mathbf{H}_{m, 1} \mathbf{p}_{m}\right|^{2} \geq\left|\mathbf{v}_{m, 2}^{H} \mathbf{H}_{m, 2} \mathbf{p}_{m}\right|^{2}$, we can verify that $\frac{\partial R_{m}^{\text {sum }}}{\partial \Omega_{m, 1}} \geq 0$. This means that the sum rate grows with $\Omega_{m, 1}$. Thus, to maximize the sum rate, as much power as possible should be allocated to the strong user $(m, 1)$, while allocating enough power to the weak user, to satisfy its QoS requirement. This completes the proof.

Based on Lemma 1, we have

$$
\log _{2}\left(1+\frac{\rho \Omega_{m, 2}\left|\mathbf{v}_{m, 2}^{H} \mathbf{H}_{m, 2} \mathbf{p}_{m}\right|^{2}}{\rho \Omega_{m, 1}\left|\mathbf{v}_{m, 2}^{H} \mathbf{H}_{m, 2} \mathbf{p}_{m}\right|^{2}+1}\right)=R_{m, 2}^{\min }
$$

Considering that $\Omega_{m, 1}+\Omega_{m, 2}=\beta$, the optimal power allocation within a cluster is

$$
\begin{aligned}
& \Omega_{m, 1}=\frac{\beta}{2^{R_{m, 2}^{\min }}}-\frac{2^{R_{m, 2}^{\min }}-1}{2^{R_{m, 2}^{\min } \rho\left|\mathbf{v}_{m, 2}^{H} \mathbf{H}_{m, 2} \mathbf{p}_{m}\right|^{2}},} \\
& \Omega_{m, 2}=\beta-\Omega_{m, 1} .
\end{aligned}
$$

Let us now discuss step two, that is, how to allocate power across the clusters. An intuitive solution could be based on iteration, where in each step some power is allocated to a cluster that needs the smallest amount of power to increase its sum rate with a unit. Along these lines, we introduce the following lemma.

Lemma 2: Denote the rate for user $(m, 1)$ as $R_{m, 1}^{\mathrm{ini}}$. Then, the extra power needed to increase the sum rate by $\Delta R$ is given by

$$
\Delta P_{m}=\left(2^{\Delta R}-1\right) P_{\max } \Phi_{m},
$$

with coefficient

$$
\Phi_{m}=\frac{2^{R_{m, 1}^{\mathrm{ini}}+R_{m, 2}^{\min }}}{\rho\left|\mathbf{v}_{m, 1}^{H} \mathbf{H}_{m, 1} \mathbf{p}_{m}\right|^{2}} .
$$

Proof: According to (7) and (9), the power coefficients can be expressed as the following with regard to the achievable rate:

$$
\begin{aligned}
\Omega_{m, 1} & =\frac{2^{R_{m, 1}^{\mathrm{ini}}}-1}{\rho\left|\mathbf{v}_{m, 1}^{H} \mathbf{H}_{m, 1} \mathbf{p}_{m}\right|^{2}} \\
\Omega_{m, 2} & =\frac{\left(2^{R_{m, 2}^{\text {min }}}-1\right)\left(1+\Omega_{m, 1} \rho\left|\mathbf{v}_{m, 2}^{H} \mathbf{H}_{m, 2} \mathbf{p}_{m}\right|^{2}\right)}{\rho\left|\mathbf{v}_{m, 2}^{H} \mathbf{H}_{m, 2} \mathbf{p}_{m}\right|^{2}} \\
& =\frac{2^{R_{m, 2}^{\min }}-1}{\rho\left|\mathbf{v}_{m, 2}^{H} \mathbf{H}_{m, 2} \mathbf{p}_{m}\right|^{2}}+\frac{\left(2^{R_{m, 1}^{\mathrm{in}}}-1\right)\left(2^{R_{m, 2}^{\min }}-1\right)}{\rho\left|\mathbf{v}_{m, 1}^{H} \mathbf{H}_{m, 1} \mathbf{p}_{m}\right|^{2}} .
\end{aligned}
$$

Due to Lemma 1, the power within each cluster should be allocated such that the weak users' QoS is just satisfied. Therefore, when the remaining power is added, only the rate of the strong user will change, while that of the weak user remains fixed. When there is a sum rate increment $\Delta R$, it is only added to the strong user, i.e., $R_{1}$ changes from $R_{m, 1}^{\text {ini }}$ to $R_{m, 1}^{\mathrm{ini}}+\Delta R$. Recall, that the required sum power at cluster $m$ is $P_{m}=P_{\max }\left(\Omega_{m, 1}+\Omega_{m, 2}\right)$. We can update the required sum power by substituting $R_{m, 1}^{\text {ini }}$ with $R_{m, 1}^{\text {ini }}+\Delta R$ in (19). Subtracting the initial sum power from the updated one we get (18).

We observe that the required power increment does not depend on the channel gain of the weak user, only on its QoS requirement. In addition, the smaller $\Phi_{m}$ is, the less power is needed for the $\Delta R$ rate increase. Thus, during each iteration, the cluster with the smallest $\Phi_{m}$ should be selected for receiving the extra power.

Based on these findings, we propose a water-filling algorithm to solve the problem (10). Water-filling has been applied for power allocation among individual users in e.g. [9], here we construct a water-filling algorithm for power allocation across as well as within the clusters.

First, an initial power allocation is performed to satisfy each weak user, resulting $R_{m, 2}=R_{m, 2}^{\min }$ and $R_{m, 1}=0$.

For each cluster $m$, we consider $\frac{P_{\max } 2_{m, 2}^{R_{m}^{\min }}}{\rho\left|\mathbf{v}_{m, 1}^{H} \mathbf{H}_{m, 1} \mathbf{p}_{m}\right|^{2}}$ as the initial water level. Moreover, we introduce an axillary variable $\lambda$ as the final water level. Consider cluster $m$. If $\lambda \leq \frac{P_{\max } 2^{R_{m, 2}^{\min }}}{\rho\left|\mathbf{v}_{m, 1}^{H} \mathbf{H}_{m, 1} \mathbf{p}_{m}\right|^{2}}$, then the cluster does not receive any 
additional power. Otherwise, it receives extra power to reach the final water level, i.e.,

$$
\lambda=\frac{P_{\max } 2^{R_{m, 2}^{\min }+\Delta R_{m}}}{\rho\left|\mathbf{v}_{m, 1}^{H} \mathbf{H}_{m, 1} \mathbf{p}_{m}\right|^{2}}=2^{\Delta R_{m}} \frac{P_{\max } 2^{R_{m, 2}^{\min }}}{\rho\left|\mathbf{v}_{m, 1}^{H} \mathbf{H}_{m, 1} \mathbf{p}_{m}\right|^{2}}
$$

where $\Delta R_{m}$ is the rate increment. According to Lemma 2, the required extra power can be expressed as

$$
\begin{aligned}
\Delta P_{m} & =\left(2^{\Delta R_{m}}-1\right) \frac{P_{\max } 2^{R_{m, 2}^{\min }}}{\rho\left|\mathbf{v}_{m, 1}^{H} \mathbf{H}_{m, 1} \mathbf{p}_{m}\right|^{2}} \\
& =\lambda-\frac{P_{\max } 2^{R_{m, 2}^{\min }}}{\rho\left|\mathbf{v}_{m, 1}^{H} \mathbf{H}_{m, 1} \mathbf{p}_{m}\right|^{2}} .
\end{aligned}
$$

Considering both cases, the required power for cluster $m$ becomes

$$
\Delta P_{m}=\left[\lambda-\frac{P_{\max } 2^{R_{m, 2}^{\min }}}{\rho\left|\mathbf{v}_{m, 1}^{H} \mathbf{H}_{m, 1} \mathbf{p}_{m}\right|^{2}}\right]^{+}
$$

and the resulting rate increment of user $(m, 1)$ is

$R_{(m, 1)}=\Delta R_{m}=\left[\log _{2}(\lambda)-\log _{2}\left(\frac{P_{\max } 2^{R_{m, 2}^{\min }}}{\rho\left|\mathbf{v}_{m, 1}^{H} \mathbf{H}_{m, 1} \mathbf{p}_{m}\right|^{2}}\right)\right]^{+}$

This provides closed form solution to problem (10), once $\lambda$ is known.

To determine $\lambda$, we consider that the sum power over all clusters should satisfy

$$
\sum_{m=1}^{M}\left[\lambda-\frac{P_{\max } 2^{R_{m, 2}^{\min }}}{\rho\left|\mathbf{v}_{m, 1}^{H} \mathbf{H}_{m, 1} \mathbf{p}_{m}\right|^{2}}\right]^{+}=P_{\max }-P_{\text {req }}
$$

The left part of the above function is a piecewise linear function, and grows monotonically with $\lambda$. Thus, the above equation admits a unique value of $\lambda$, that can be found iteratively as given in Algorithm 1 .

Theorem 1: The proposed C-NOMA algorithm maximizes the sum rate of the system, when all weak users can be admitted.

Proof: Assume that we have obtained the solution via the proposed PA algorithm i.e., $\lambda$ and $\Delta P_{m}$ are known for all $m$. Now, we shift some power $\Delta p$ between two clusters whose final water level is $\lambda$. Denote the two clusters with $q$ and $n$. In the proposed PA scheme, for the $q$ th cluster, we have

$$
\begin{aligned}
\Delta R_{q}= & \log _{2}(\lambda)-\log _{2}\left(\frac{P_{\max } 2^{R_{q, 2}^{\min }}}{\rho\left|\mathbf{v}_{q, 1}^{H} \mathbf{H}_{q, 1} \mathbf{p}_{q}\right|^{2}}\right) \\
= & \log _{2}\left(\Delta P_{q}+\frac{P_{\max } 2^{R_{q, 2}^{\min }}}{\rho\left|\mathbf{v}_{q, 1}^{H} \mathbf{H}_{q, 1} \mathbf{p}_{q}\right|^{2}}\right) \\
& -\log _{2}\left(\frac{P_{\max } 2^{R_{q, 2}^{\min }}}{\rho\left|\mathbf{v}_{q, 1}^{H} \mathbf{H}_{q, 1} \mathbf{p}_{q}\right|^{2}}\right) .
\end{aligned}
$$

After shifting $\Delta p$ power between the two clusters, we get

$$
\begin{aligned}
\Delta R_{q}^{\prime}= & \log _{2}\left(\Delta P_{q}+\Delta p+\frac{P_{\max } 2^{R_{q, 2}^{\min }}}{\rho\left|\mathbf{v}_{q, 1}^{H} \mathbf{H}_{q, 1} \mathbf{p}_{q}\right|^{2}}\right) \\
& -\log _{2}\left(\frac{P_{\max } 2^{R_{q, 2}^{\min }}}{\rho\left|\mathbf{v}_{q, 1}^{H} \mathbf{H}_{q, 1} \mathbf{p}_{q}\right|^{2}}\right) \\
= & \log _{2}(\lambda+\Delta p)-\log _{2}\left(\frac{P_{\max } 2^{R_{q, 2}^{\min }}}{\rho\left|\mathbf{v}_{q, 1}^{H} \mathbf{H}_{q, 1} \mathbf{p}_{q}\right|^{2}}\right) .
\end{aligned}
$$

Likewise, we can attain the expressions for $\Delta R_{n}$ and $\Delta R_{n}^{\prime}$, respectively. Accordingly, the sum rate difference $\Delta R_{\text {sum }}=$ $\Delta R_{q}+\Delta R_{n}-\Delta R_{q}^{\prime}-\Delta R_{n}^{\prime}$ becomes

$$
\begin{aligned}
\Delta R_{\text {sum }} & =\log _{2}(\lambda)+\log _{2}(\lambda)-\log _{2}(\lambda+\Delta p)-\log _{2}(\lambda-\Delta p) \\
& =\log _{2}\left(\lambda^{2}\right)-\log _{2}\left[\lambda^{2}-(\Delta p)^{2}\right]>0 .
\end{aligned}
$$

This proves that shifting power between two clusters whose water level is $\lambda$ leads to a decreased sum rate. Following the same procedure, it can be proved that the same holds when shifting some power from one cluster with water level equal to $\lambda$ to another cluster with water level exceeding $\lambda$. This completes the proof.

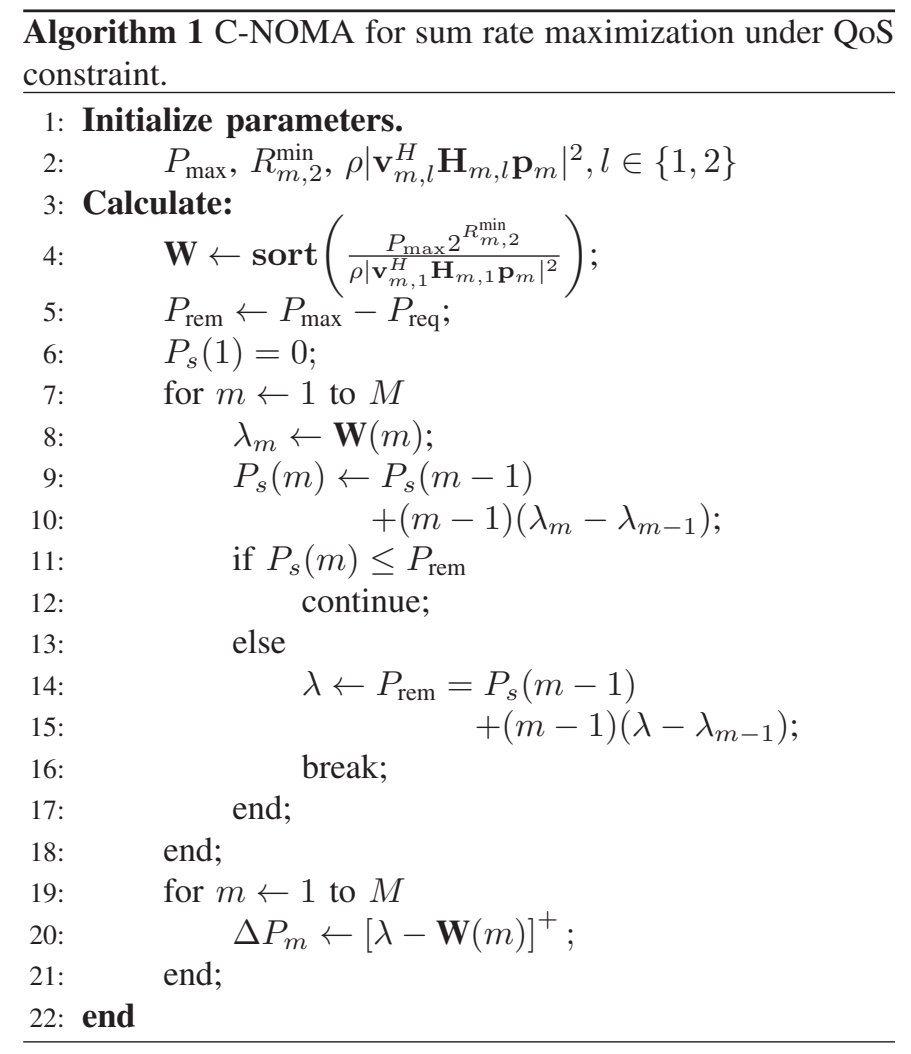

\section{B. C-NOMA admission algorithm when (10) is infeasible}

In this case, problem (11) is addressed, and user admission is conducted as follows: the weak users are admitted one by one following the ascending order of the power required for fulfilling their QoS constraint. This power can be calculated according to (13). The admission is stopped if the next user cannot be satisfied with the remaining, unallocated power. 


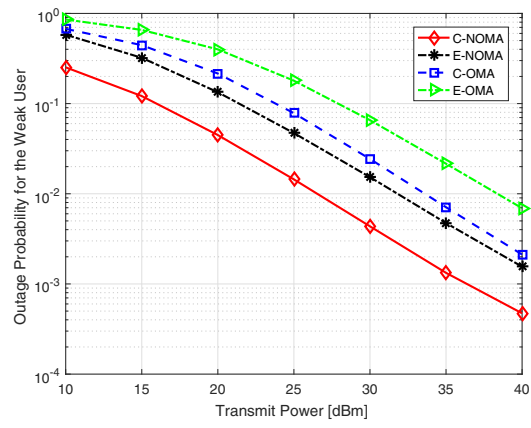

(a)

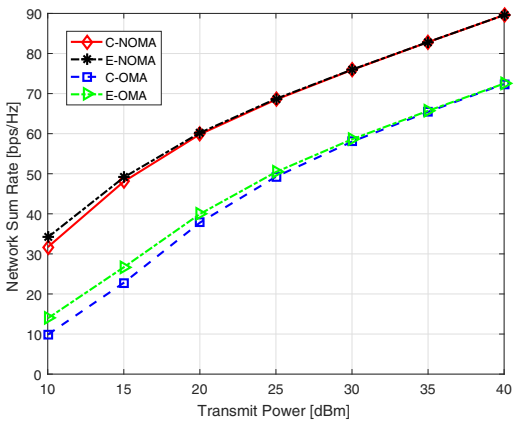

(b)

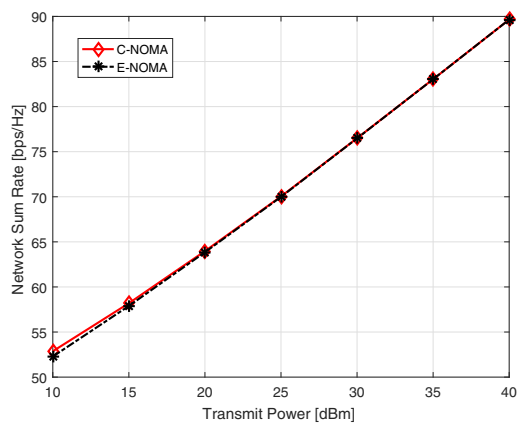

(c)

Fig. 1: Outage probability and network sum rate versus the transmit power $P_{m}$ : a) outage probability of the weak users; b) network sum rate; and c) network sum rate when all weak users are admitted.

Theorem 2: The proposed user admission scheme maximizes the number of admitted users.

Proof: During each iteration, the user with the minimum required power is admitted. As the total power is fixed, the less the required power for each user, the larger the number of admitted users. Thus, the proposed scheme maximizes the number of admitted users.

\section{Complexity analysis}

When (10) is feasible, the sum rate maximization problem takes $O\left(M \log _{2}(M)\right)$ to obtain the optimal results according to Algorithm 1; $O\left(M \log _{2}(M)\right)$ comes from the sorting algorithm in line 4. When (10) is infeasible, the user admission algorithm also takes $O\left(M \log _{2}(M)\right)$ steps, which comes from the ordering of the required power for the weak users. Thus, the overall complexity is $O\left(M \log _{2}(M)\right)$.

\section{Baseline algorithms}

To show the benefits of C-NOMA, we use the CR-PA solution in [8], denoted by E-NOMA as the baseline algorithm. As the main difference, E-NOMA allocates equal total power for each cluster. Then, for each cluster, it determines whether QoS of the weak user can be satisfied or not. If so, the power is allocated such that the QoS of the weak user is just satisfied, that is, rate maximization in the cluster is achieved, according to Lemma 1. Otherwise, the weak user receives all the power.

In addition, to show the superiority of NOMA over OMA, we also consider OMA with equal power for each cluster as well as cross-cluster PA (denoted as E-OMA and C-OMA, respectively). When equal power is allocated to each cluster for OMA, it is first decided whether the weak user can be admitted or not. If so, the power is allocated to the weak user such that its QoS is satisfied, and water-filling technique is applied to allocate the remaining power between the two users [9]. Otherwise, all the power is assigned to the weak user. When cross-cluster PA is considered, and all weak users can be admitted, then first power is allocated to the users such that each weak user's QoS is satisfied. After that, waterfilling technique is applied among all the users to allocate the remaining power. If not all weak users can be admitted, then they are arranged on descending order of their channel gains, and are admitted one by one until all power is consumed.

TABLE I: Simulation Parameters.

\begin{tabular}{c|c}
\hline Parameter & Value \\
\hline \hline Number of antennas & $M=4, N=3$ \\
\hline Channel bandwidth & $10[\mathrm{MHz}]$ \\
\hline Thermal noise density & $-174[\mathrm{dBm} / \mathrm{Hz}]$ \\
\hline Path-loss model & $114+38 \log _{10}(d), d$ in kilometer \\
\hline
\end{tabular}

\section{Performance Evaluation}

We evaluate the performance of the sum rate maximizing PA, with parameter values given in Table I. Four strong users are placed randomly uniformly within $d_{1}=50 \mathrm{~m}$ distance from the BS, while four weak users are placed within a range of $\left(d_{1}, d_{2}\right)$, with a default value of $d_{2}=100 \mathrm{~m}$. Weak and strong users are paired randomly to form four NOMA clusters. Matrix $\mathbf{H}$ is generated according to the path loss model and the considered Rayleigh fading. Weak users have a minimum rate requirement of $3 \mathrm{bps} / \mathrm{Hz}$. We consider power levels, where the weak users can be accepted with reasonable probability, and present results averaged over $10^{5}$ random configurations.

On Fig. 1a, we show the outage probability of the weak users, that is, the probability that they cannot be admitted, under increasing transmit power $P_{\max }$. Clearly, NOMA outperforms OMA in terms of the outage probability of the weak user. In addition, for both NOMA and OMA, cross-cluster PA achieves lower outage probability than equal power PA. Specifically, C-NOMA halves the outage probability of the weak users, compared to E-NOMA.

Fig. 1b compares the sum rates under the different PA algorithms. As expected, NOMA achieves significantly higher sum rates than OMA, especially in the low transmit power regime. There is no significant difference in the sum rates of the equal power and cross-cluster PA solutions. Under low transmit power, cross-cluster PA attains slightly lower sum rate compared to equal power PA ( $7 \%$ loss when the transmit power is $10 \mathrm{dBm}$ ), since it admits a higher number of weak users. Note that this does not contradict Theorem 1, since it 

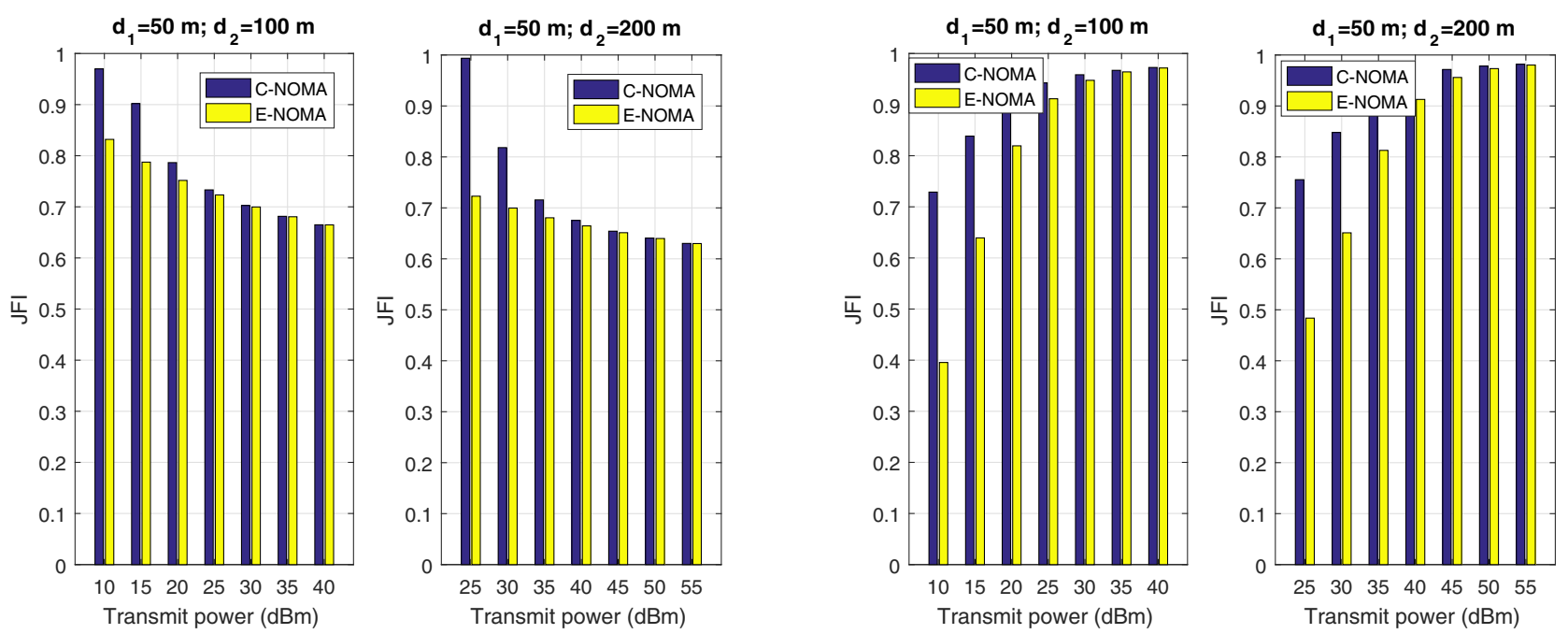

(a) User fairness

(b) Cluster fairness

Fig. 2: Fairness of C-NOMA and E-NOMA under different $d_{2}$ distance values, considered a) for weak and strong users, and b) for the sum rate of the clusters.

holds when all the weak users are admitted. Fig. 1c shows the average sum rate considering only the cases when all weak users are admitted, verifying Theorem 1.

We evaluate the resulting fairness of E-NOMA and CNOMA in Fig. 2. We measure user fairness as the averaged Jain's fairness index (JFI) [10]. Fig. 2a shows the JFI of the achieved rate between the weak and strong user in the cluster, while Fig. $2 \mathrm{~b}$ considers the fairness of the sum rates in the four clusters. We show results for $d_{2}=100 \mathrm{~m}$ and $d_{2}=200 \mathrm{~m}$. For both scenarios, the system behaviour is similar. C-NOMA achieves higher user fairness and cluster fairness than ENOMA, the difference is significant when considering cluster fairness under low transmit power. For both schemes, as the transmit power increases, user fairness decreases, since strong users get higher and higher rates. Cluster fairness grows with increasing power, as the sum rates of the clusters saturate.

\section{CONCLUSION}

In this paper, we considered clustered MIMO-NOMA downlink transmission, where fairness is facilitated through a QoS constraint for users with weak channel condition. We studied the effects of the power allocation within and across the NOMA clusters, and proposed C-NOMA that allocates transmit power across the NOMA clusters through a water-filling procedure. We proved that C-NOMA maximizes the network sum rate under the QoS constraint, when all weak users can be satisfied. The numerical results demonstrated that through the rate maximizing cross-cluster power allocation, C-NOMA can decrease the outage probability of the weak users by half, without affecting the achieved sum rate in the network.

\section{REFERENCES}

[1] Y. Saito, Y. Kishiyama, A. Benjebbour, T. Nakamura, A. Li, and K. Higuchi, "Non-orthogonal multiple access (NOMA) for cellular future radio access," in Proc. IEEE Veh. Technol. Conf., Dresden, Germany, Jun. 2013.

[2] L. Dai, B. Wang, Y. Yuan, S. Han, C. 1. I, and Z. Wang, "Non-orthogonal multiple access for $5 \mathrm{G}$ : Solutions, challenges, opportunities, and future research trends," IEEE Commun. Mag., vol. 53, no. 9, pp. 74-81, Sep. 2015.

[3] Z. Ding, Y. Liu, J. Choi, Q. Sun, M. Elkashlan, C. L. I, and H. V. Poor, "Application of non-orthogonal multiple access in LTE and 5G networks," IEEE Commun. Mag., vol. 55, no. 2, pp. 185-191, February 2017.

[4] L. Lei, D. Yuan, C. K. Ho, and S. Sun, "Power and channel allocation for non-orthogonal multiple access in $5 \mathrm{G}$ systems: Tractability and computation," IEEE Trans. Wireless Commun., vol. 15, no. 12, pp. 85808594, Dec. 2016.

[5] J. A. Oviedo and H. R. Sadjadpour, "A fair power allocation approach to NOMA in multiuser SISO systems," IEEE Trans. Veh. Technol., vol. 66, no. 9, pp. 7974-7985, Sept 2017.

[6] Z. Ding, P. Fan, and H. V. Poor, "Impact of user pairing on 5G nonorthogonal multiple-access downlink transmissions," IEEE Trans. Veh. Technol., vol. 65, no. 8, pp. 6010-6023, Aug. 2016.

[7] Z. Ding, F. Adachi, and H. V. Poor, "The application of MIMO to nonorthogonal multiple access," IEEE Trans. Wireless Commun., vol. 15, no. 1, pp. 537-552, Jan. 2016.

[8] Z. Ding, R. Schober, and H. V. Poor, "A general MIMO framework for NOMA downlink and uplink transmission based on signal alignment," IEEE Trans. Wireless Commun., vol. 15, no. 6, pp. 4438-4454, Jun. 2016.

[9] D. Tse and P. Viswanath, Fundamentals of Wireless Communication. Cambridge, UK: Cambridge University Press, 2005.

[10] R. Jain, D. M. Chiu, and W. Hawe, "A quantitative measure of fairness and discrimination for resource allocation in shared computer system," DEC Research Report TR-301, 1984. 